\title{
Effect of Moderate Physical Exercise on Autonomic Balance in Irritable Bowel Syndrome
}

\author{
Karma Tenzin ${ }^{1}$, Noorzahan Begum², Sultana Ferdousi ${ }^{3}$
}

\begin{abstract}
Background: Altered autonomic balance has been noted in Irritable bowel syndrome Regular physical exercise may cause restoration of autonomic balance in health and disease. Objective: To assess the effect of brisk walking on the autonomic balance by analysis of heart rate variability in patients with Irritable bowel syndrome (IBS). Methods: This prospective study was conducted in the Department of Physiology, Bangabandhu Sheikh Mujib Medical University (BSMMU) in 2013.Seventy seven male IBS patients aged 20-50 years, were enrolled from Gastroenterology OPD, BSMMU, Dhaka. Twenty eight healthy sedentary male with similar age were control. HRV measures were recorded once prior to exercise and then after 3 months of brisk walking. For assessing autonomic balance, LF/HF ratio and Max/Min RR was evaluated by Polyrite D machine and software. ANOVA, Independent sample t-test and paired t-test were used for statistical analysis. Results: The pre-exercise mean values of LF/HF ratio were significantly higher $(\mathrm{p}<0.001)$ in all IBS patients compared to those of control. The post exercise LF/HF ratio were significantly lower $(\mathrm{p}<0.05)$ in all IBS patients compared to their pre-exercise values. Conclusion: This study concluded that the sympathovagal balance was towards sympathetic predominance in IBS and regular moderate physical exercise may shift the balance towards parasympathetic predominance in them.
\end{abstract}

Key words: Irritable bowel syndrome, sympathovagal balance, Male, Physical exercise, Heart rate variability.

J Bangladesh Soc Physiol. 2015, June; 10(1): 6-10 For Authors Affiliation, see end of text.

http://www.banglajol.info/index.php/JBSP

\section{Introduction}

I BS is an intermittent or continuously occurring chronic functional gastrointestinal disorder that is associated with abdominal pain, bloating and bowel disturbance in the absence of any organic cause ${ }^{1}$.

In accordance with the predominant symptoms present, IBS has been classified as Diarrhoea predominant irritable bowel syndrome (IBS-D), Constipation predominant IBS (IBS-C) and Irritable bowel syndrome alternating type (IBS-A) ${ }^{2}$.

Received March 2014;

Accepted October 2014
Though pathophysiology of IBS still remains unclear, recent works suggested that it results from a disorder of the brain gut axis, altered visceral sensitivity and altered bowel motility ${ }^{3}$.

There are reports of autonomic dysfunction in IBS characterized by increased sympathetic and decreased parasympathetic activity ${ }^{4}$ and also vagal dysfunction in IBS-C and sympathetic dysfunction in IBS-D ${ }^{2}$.

Regular physical exercise has demonstrated improvement in autonomic nerve function in both 
health and in various clinical conditions such as myocardial infarction, rheumatoid arthritis, heart failure, coronary artery disease, type II diabetes mellitus, chronic renal failure, obesity and menopause $\mathrm{e}^{5-10}$.

Though, several studies reported improvement of autonomic nervous activities under regular physical exercise but few failed to show any significant change. ${ }^{11-13}$

According to task force LF/HF ratio evaluated in power spectral analysis of HRV is an important marker for sympathovagal balance or autonomic balance at any instant. ${ }^{14-15}$.

Tillish et $\mathrm{al}^{16}$. and Nayem et $\mathrm{al}^{15}$ reported higher $\mathrm{LF} / \mathrm{HF}$ ratio in sedentary IBS than sedentary control whereas lower LF/HF ratio was noted in hypertensive patients after physical exercise. ${ }^{17}$

Lower LF/HF ratio was also noted in physically active subjects compared to sedentary control ${ }^{5-}$ 6 , but few studies failed to present any effect of physical exercise on the LF/HF ratio ${ }^{18-19}$.

Though, shift of sympathovagal balance towards parasympathetic dominance was induced by physical exercise in healthy sedentarys ${ }^{5-6}$ but the effect of physical exercise on $\mathrm{LF} / \mathrm{HF}$ ratio was not studied in sedentary IBS patients.

Therefore, this study has been designed to evaluate the effect of moderate physical exercise by brisk walk on the autonomic balance in patients with IBS.

\section{Methods}

This prospective interventional study was conducted in the Department of Physiology, Bangabandhu Sheikh Mujib Medical University (BSMMU), Dhaka from January to December 2013. The protocol of this study was approved by Institutional Review Board of Bangubandhu Sheikh Mujib Medial University. For this purpose, 77 male patients aged $20-50$ years with Irritable bowel syndrome were enrolled for the study by random sampling from Gastroenterology OPD, BSMMU. 28 male healthy controls with similar age and BMI were recruited through personal contact.

According to the predominant symptom present, all IBS patients were divided into 3 subgroups, these included 24 diarrhea predominant IBS (IBSD), 26 constipation predominant IBS (IBS-C) and 27 altered bowel habit type IBS (IBS-A) patients. 28 apparently healthy sedentary male were enrolled as control. History of chronic illnesses such as heart disease, diabetes mellitus, hypertension, chronic renal failure, thyroid disorders and smoking were excluded and also history of regular physical exercise were ruled out in all selected subjects.

Informed written consent was obtained from all the study participants. Subsequently, a detail family and medical histories and also physical activity status were recorded.

For HRV recording, the subjects were prepared from one day prior to the test. They were advised to take their meal by 9:00 p.m and have sound sleep and avoid any physical or mental stress, and also sedative medications. In addition, they were advised to take light breakfast in the morning without tea and coffee.

All the examinations were done in the Autonomic Nerve Function Test Laboratory in the Department of Physiology, BSMMU. For the purpose of HRV recording, the subject was given bed rest for 15-20 minutes, after which 5 minutes ECG recording was carried out for HRV by polyrite D machine.

On completion of baseline HRV recording, the patients performed brisk walking for 3 months. For this purpose, patients were trained on how to perform brisk walking at a rate of 130 steps per minute for 30 to 45 minutes, for 3 to 5 days in a week for a period of 3 months duration ${ }^{1,20-21}$. However, this exercise intervention was not applied to the control group.

The LF/HF ratio represents the sympathovagal balance and max/min RR represent variability. 
these variables were analyzed before exercise and similarly after 3 months of moderate physical exercise. For statistical analysis, ANOVA, independent sample t-test and paired sample t-test were performed.

\section{Results}

The pre-exercise mean values of systolic blood pressure (SBP), diastolic blood pressure (DBP) and pulse rate (PR) were found significantly higher in all IBS patients compared to those of control.(Table I)
Pre-exercise mean values of $\mathrm{LF} / \mathrm{HF}$ ratio were higher $(\mathrm{p}<0.001)$ in all IBS groups than those of the control. However, No significant differences of this parameter was observed between subgroups of IBS patients. (Table II). In addition, pre exercise values of max/min ratio of RR interval were significantly different between IBS D and IBS C and also IBS C vs IBS A.

Post exercise mean values of $\mathrm{LF} / \mathrm{HF}$ ratio were lower $(\mathrm{p}<0.01)$ in all IBS patients compared to their values in corresponding pre-exercise IBS groups.

Table I: General Characteristics in different groups ( $\mathrm{n}=105)$

\begin{tabular}{lcccc}
\hline Parameters & Control & IBS-D & IBS-C & IBS-A \\
\hline Age & $32 \pm 1.26$ & $32 \pm 1.36$ & $29 \pm 1.36$ & $32 \pm 1.37$ \\
BMI & $22.8 \pm 0.29$ & $22.2 \pm 0.48$ & $21.8 \pm 0.43$ & $22.3 \pm 0.44$ \\
SBP & $114 \pm 1.55$ & $120 \pm 1.63^{*}$ & $120 \pm 1.96^{*}$ & $120 \pm 1.79^{*}$ \\
DBP & $71 \pm 1.25$ & $75 \pm 1.59^{*}$ & $77 \pm 1.66^{*}$ & $76 \pm 1.24^{*}$ \\
PR & $74 \pm 0.96$ & $86 \pm 1.64^{* *}$ & $84 \pm 1.23^{* *}$ & $86 \pm 1.48^{* *}$ \\
\hline
\end{tabular}

Values in means \pm SE for Diarrhoea predominant Irritable bowel syndrome (IBS-D), Constipation predominant Irritable bowel syndrome (IBS-C) \& altered bowel habit type of Irritable bowel syndrome. $* * \mathrm{p}<0.01 \& \mathrm{p}^{*} \mathrm{p}$ $0.05(*=$ Significant difference IBS versus control)

Table II: Pre-exercise and post exercise LF/HF ratio and Max/min RR of HRV in different groups $(\mathrm{n}=105)$

\begin{tabular}{|c|c|c|c|c|c|c|c|}
\hline \multirow[t]{3}{*}{ Parameters } & \multirow{3}{*}{$\begin{array}{l}\text { Control } \\
\text { No exercise } \\
\text { Baseline }\end{array}$} & \multirow{2}{*}{\multicolumn{2}{|c|}{$\begin{array}{l}\text { IBS-D } \\
\text { Exercise }\end{array}$}} & \multirow{2}{*}{\multicolumn{2}{|c|}{$\begin{array}{c}\text { IBS-C } \\
\text { Exercise }\end{array}$}} & \multirow{2}{*}{\multicolumn{2}{|c|}{$\begin{array}{c}\text { IBS-A } \\
\text { Exercise }\end{array}$}} \\
\hline & & & & & & & \\
\hline & & Pre & Post & Pre & post & Pre & Post \\
\hline \multirow[t]{2}{*}{$\mathrm{LF} / \mathrm{HF}$} & $1.57 \pm$ & $2.64 \pm$ & $1.79 \pm$ & $2.82 \pm$ & $1.77 \pm$ & $2.84 \pm$ & $1.80 \pm$ \\
\hline & 0.06 & $0.25^{* * *}$ & $0.07^{‘} \&^{‘} \ldots$ & $0.26^{* * *}$ & $0.07^{‘} \&^{‘} \ldots$ & $0.19^{* * *}$ & $0.09^{\prime} \& ` \& ` \&$ \\
\hline Max/Min RF & $1.79 \pm 0.11$ & $2.04 \pm 0.16$ & $1.82 \pm 0.11$ & $1.66 \pm 0.09$ & $1.74 \pm 0.08$ & $1.57 \pm 0.07^{¥ ¥}$ & $1.73 \pm 0.07$ \\
\hline
\end{tabular}

Values are means $\pm \mathrm{SE}$, Statistical analysis were performed by independent sample t-test and paired ttest. Diarrhoea predominant Irritable bowel syndrome (IBS-D), Constipation predominant Irritable bowel syndrome (IBS-C) \& altered bowel habit type of Irritable bowel syndrome(IBS -A). $*^{*}=$ control vs preexercise; ‘ $\&=$ pre vs post exercise $\mathbb{I =}$ Pre exercise D vs C; $¥=$ Pre exercise C vs A) $8 * * * \mathrm{p}<0.1$ ' $\&$ ‘ $\& \& \&$ $<90.001, \mathrm{p}<0.05$ वा $\mathrm{p}<0.05$ 


\section{Discussion}

Results of this prospective study showed the changes in autonomic balance in IBS patients and reversal of this altered balance brought about by moderate physical exercise for 3 months causing shifting of sympathovagal balance from sympathetic predominance to parasympathetic predominance and decrease the symptoms in patients with irritable bowel syndrome.

In this study pre-exercise LF/HF ratio were significantly higher in all IBS patients and these altered baseline $\mathrm{LF} / \mathrm{HF}$ ratio indicate change of autonomic balance towards sympathetic dominance in all IBS patients ${ }^{15,16,22}$.

The significant increase in post exercise values of these parameters are consistent with other studies conducted to observe the effect of physical activity on LF/HF ratio but in healthy subjects $5-6,17$.It is also notable that these parameters are not significantly different among the subtypes irrespective of pre or post exercise.

It is obvious from the results of this study that moderate physical exercise caused shifting of the sympathovagal balance in IBS patients towards more parasympathetic predominance which was previously sympathetic dominance before exercise, supported by significant fall of LF/HF ratio in post exercise period. This is consistent with various investigators who proposed that moderate physical exercise is associated with either increased vagal tone or decreased sympathetic modulation of heart rate in both healthy and diseased conditions ${ }^{5-6,17}$.

Physical exercise reduces the stress by influencing brain plasticity ${ }^{1}$ which results in higher parasympathetic activity and improvement in cardiovagal baroreflex sensitivity. In addition, both acetylcholine and choline-acetyl transferase content in cardiac tissue may be increased, thereby parasympathetic nervous activity ${ }^{6}$. Moreover, physical exercise also might promote adjustments in cardiovascular control sites via either neural remodeling or endogenous factors like nitric oxide and angiotensin II. It has been suggested that these adaptations might cause decrease in sympathetic and increase in vagal component in cardiac control to improve cardiac autonomic balance ${ }^{23-25}$. The lack of discrimination of exercise induce change in autonomic balance among different sub type of IBS patients suggests symptoms based characteristics are independent of autonomic modulation.

\section{Conclusion}

This study concludes that male patients with all the three subgroups of IBS had noticeable sympathovagal balance with sympathetic predominance, however moderate physical exercise could shift the balance towards parasympathetic dominance by increasing cardiac parasympathetic activity and decreasing the cardiac sympathetic activity.

Conflict of Interest: None

\section{Author affiliations}

*1. Karma Tenzin, Lecturer, Dept of basic science, Khesar Gyalpo University of Medical Sciences of Bhutan,Thimpu,Bhutan.Email:karmatenzin9@gmail.com

2. Noorzahan Begum, Professor, Dept of Physiology, Bangabandhu Sheikh Mujib Medical University, Dhaka. Email:noorzahan52@gmail.com

3. Sultana Ferdousi, Associate Professor, Dept of Physiology, Bangabandhu Sheikh Mujib Medical University, Dhaka Email: sferdousiratna@gmail.com

*For correspondence

\section{References}

1. Johannesson E, Simren M, Strid H, Bajor A, Sadik R. Physical activity improves symptoms in irritable bowel syndrome: a rantomized controlled trial. Am J Gastroenterol 2011; 106: 915-22.

2. Heitkemper M, Jarrett M, Cain KC, Burr R, Levy RL, Feld A, Hertig V. Autonomic nervous system function in women with irritable bowel syndrome. Dig Dis Sci 2001; 46(6): 1276-84.

3. Barbara G, De Giorgio R, Stanghellini V, Cremon C, Salvioli B, Corrinaldesi R. New pathophysiological mechanisms in irritable bowel syndrome. Aliment Pharmacol Ther 2004; 20(2): 1-9.

4. Adeyemi EOA, Desai KD, Towsey M, Ghista D. Characterization of autonomic dysfunction in patients with irritable bowel syndrome by means of 
heart rate variability studies. Am J Gastroenterol 1999; 94(3): 945-50.

5. Alom MM, Begum N, Naher LAD, Faruky SB, Akter N. Study on heart rate variability in adolescent male athletes by time domain (Short-Term) method. The ORION Medical Journal 2010; 33(1): 722-25

6. Mithun S, Begum N, Ferdousi S, Begum S, Ali T. Time domain measures of heart rate variability in heavy workers. J Bangladesh Soc Physiol 2009; 6(2):77-83.

7. Malfatto G, Facchini M, Bragato R, Branzi G, Sala L, Leonetti G. Short and long-term effects of exercise training on the tonic autonomic modulation of heart rate variability after myocardial infarction. Eur Heart J 1996; 17: 532-538.

8. Iellamo F, Pizzinelli P, Massaro M, Raimondi G, Peruzzi G, Legramante JM. Muscle metaboreflex contribution to sinus node regulation during static exercise: Insight from spectral analysis of heart rate variability. Circulation 2000; 100: 27-32.

9. Pagkalos M, Koutlianos N, Kouidi E, Pagakalos E, Mandroukas K, Deligiannis A. Heart rate variability modifications following exercise training in type 2 diabetic patients with definite cardiac autonomic neuropathy. Br J Sports Med 2008; 42: 47-54.

10. Bonaduce D, Petretta M, Cavallaro V, Apicella C, Ianniciello A, Romano M, Breglio R, Marciano $\mathrm{F}$. Intensive training and cardiac autonomic control in high level athletes. Med Sci Sports Exerc 1998; 30(5): 691-96.

11. Dixon EM, Kamath MV, McCartney N, Fallen L. Neural regulation of heart rate variability in endurance athletes and sedentary controls. Cardiovasc Res 1992; 26(7): 713-719.

12. Dina C, Rebsburg JV, Ker JA, Grants CC, Fletcher L. Effect of exercise on cardiac autonomic function in females with rheumatoid arthritis. Clin Rheumatol 2012; 31: 1155-62.

13. Figueroa A, Baynard T, Fernhall B, Carhart R, Kaneley JA. Endurance training improves postexercise cardiac autonomic modulation in obese women with and without type-2 diabetes. Eur J Appl Physiol 2007; 100: 437-44.

14. Task Force of the European Society of Cardiology and the North American Society of pacing and electrophysiology. Heart Rate Variability. standards of measurement, physiological interpretation and clinical use. Circulation 1996; 93: 1043- 65.

15. Nayem M, Begum N, Ferdousi S. Assessment of Autonomic Nerve Function in patient in irritable bowel syndrome. J. Bangledesh Soc Physiol 2012; 7(1): 53-59.
16. Tillisch K, Mayer EA, Labus JS, Stains J, Chang L, Naliboff BD. Characterization of the alternating bowel habit subtype in patients with irritable bowel syndrome. Am J gastroenterol 2005; 100: 896-904.

17. Cozza IC, Sacco THRD, Mazon JH, Salgado MCO, Dutra SGV, Cesarino EJ, Souza HCD. Physical exercise improves cardiac autonomic modulation in hypertensive patients independently of angiotensin-converting enzyme inhibitor treatment. Hypertension Research 2012; 35: 82-87.

18. Costa PJ, Carvalho H, Freitas A, Ramos J, Puga N, Lomba I, Fernandes P, de Freitas F. Spectrum analysis of the variability heart rate in athletes. Rev Port Cardiol 1991;10(1): 23-8.

19. Dixon EM, Kamath MV, McCartney N, Fallen L. Neural regulation of heart rate variability in endurance athletes and sedentary controls. Cardiovasc Res 1992; 26(7): 713-19.

20. Ainsworth BE, Haskell WL, Herrmann SD, Meckes N, Bassett DR, Tudor-locke C, Greer JL, Vezina J, Whitt-Glover MC, Leon AS. 2011 Compendium of physical activities: A second update of caeds and MET Values. [Internet]. [cited 2012 Nov 10]. Available from: http://www. msse.org.

21. Miyashita M, Burns SF, Stensel DJ. Accumulating short bouts of brisk walking reduce postprandial plasma triacylglycerol concentrations and resting blood pressure in healthy young men. Am J Clin nutr 2008); 8: 1225-31.

22. Jarrett ME, Burr RL, Cain KC, Hertig V, Weisman P, Arnp M, Heitkemper MM. Anxiety and depression are related to autonomic nervous system function in women with irritable bowel syndrome. Dig Dis Sci 2003; 48(2): 386-94.

23. Marcos B A, Araujo CG. Effects of aerobic training on heart rate. Rev Bras Med E sport 2003; 9: 113-20.

24. Du N, Bai S, Oguri K, Kato Y,Matsumoto I, Kawase $\mathrm{H}$, Matsuoka T. Heart rate recovery after exercise and neural regulation of heart rate variability in 30-40 year old female marathon runners. J sport sci med 2005; 4: 9-17.

25. Marud K, Brubaker PH, Fitzgerald DM, Morgan TM, Goff DC, Soliman EZ, Eggebeen JD, Kitzman DW. Exercise training improves heart rate variability in older patients with heart failure: a randomized. Controlled, single-blinded trial. Congest heart fail 2012; 18: 192-197.

J Bangladesh Soc Physiol. 2015, June; 10(1): 6-10 